\title{
Efficacy of the Subclavian Portal Approach in Arthroscopic Repair of Isolated Subscapularis Tendon Tear
}

\author{
Seung Bum Chae, Chang Hyuk Choi, Suk-han Jung \\ Department of Orthopaedic Surgery, College of Medicine, Catholic University of Daegu, Daegu, Korea
}

Background: To evaluate the efficacy of the subclavian portal approach for the arthroscopic repair of isolated subscapularis tendon tear. Methods: We used the subclavian portal to carry out arthroscopic repair of the isolated subscapularis tendon tear. The surgery was carried out in 18 cases (average age of 53) from May 2006 to December 2009 with a mean follow-up period of 35 months. Of these cases, 13 patients had the tear in their dominant arms, 16 were male, and 12 were from traumatic ruptures with on average 7.6 months of symptom period to operation. Additional surgery, acromioplasty and subacromial debridement, were carried out on 4 cases each during the subscapularis repair. The integrity of cuff status was assessed by ultrasonographic examination at 6 months and at 1 year after operation.

Results: The initial average range of motion in forward flexion, external rotation, and internal rotation were $160^{\circ}, 50^{\circ}$ and $\mathrm{L} 4$, respectively. At the 1 year follow-up period, these improved to $160^{\circ}, 52^{\circ}$ and $\mathrm{T} 12$, respectively. The initial average functional scores were assessed by KSS, ASES, UCLA, and Constant scoring systems, which were 67, 60, 26, and 65, respectively. These scores improved progressively with time. At 3 months after operation, the scores were 74, 67, 27, and 74; at 6 months, 83, 77, 31, and 75; at 1 year, 88, 86, 32, and 79; and at the final follow-up of 35 months, 84, 92, 34, and 84.

Conclusions: In the repair of isolated subscapularis tendon tear, the subclavian portal approach provided a good angle for anchor insertion and sufficient space to manage the upper portion of the tendon tear. In turn, these provisions resulted in satisfactory clinical results.

(Clin Shoulder Elb 2014;17(1):18-24)

Key Words: Subscapularis tendon; Subclavian portal; Suture anchor

\section{Introduction}

The arthroscopic subscapularis tendon repair is one of the most difficult surgeries in arthroscopic shoulder joint surgery. First, the posterior portal is used, which does not give sufficient space or a clear view to see and carry out surgery on the anchor site of the subscapularis tendon. Second, a hemorrhage during surgery may form an edema, which makes the operation of surgical tools be trickier. Other portals are also used in existing methods to carry out subscapularis tendon repair, which are the anterior and anterolateral portals. During repair, these portals are used to pull out the subscapularis tendon and debride the footprint, after which the suture anchor is inserted to start the repair. ${ }^{1,2}$ In contrast to previous methods, our repair method uses two different portals instead of a specific one. We used the subclavian portal to carry out the interwave suture technique subcutaneously using a suture anchor and suture link, and the anterior portal for suture ligation.

\section{Methods}

The subclavian portal was used to carry out to repair of isolated subscapularis tendon tears. The surgery was carried out in 18 cases (average age of 53, range from 33-69) from May

Received August 16, 2013. Revised December 14, 2013. Accepted March 6, 2014.

Correspondence to: Chang-Hyuk Choi

Department of Orthopaedics Surgery, College of Medicine, Catholic University of Daegu, 33 Duryugonwon-ro 17-gil, Nam-gu, Daegu 705-718, Korea

Tel: +82-53-650-4276, Fax: +82-53-650-4272, E-mail: chchoi@cu.ac.kr

Financial support: None. Conflict of interests: None. 
2006 to December 2009 with a follow-up period of at least 1 year. Of these cases, 16 cases were male, 2 cases were female, and 13 cases had the tear in their dominant arms. Before the surgery, the degree of tear was assessed using MRI scan, which were then categorized into the standards made by Pfirrmann et al. ${ }^{3)}$ According to these standards, 13 cases had partial tear (slight fraying and signal alteration) in the cranial part of the subscapularis tendon, 3 cases had tear in the cranial 3/4 of the tendon, and 2 cases had complete tear (Fig. 1). Furthermore, 8 cases had mild gradient trauma and 4 had above-average trauma. Excluding the 6 non-traumatic ruptures, these 12 traumatic tears had on average 7 months of symptom period before the operation was carried out. Also, some additional surgery, acromioplasty (4 cases) and subacromial debridement (4 cases), were carried out during the subscapularis repair. The average functional scores were assessed by the Korean shoulder scoring (KSS), ASES, UCLA, and Constant scoring systems before the operation and at the following points after: 3 months, 6 months and 1 year. In
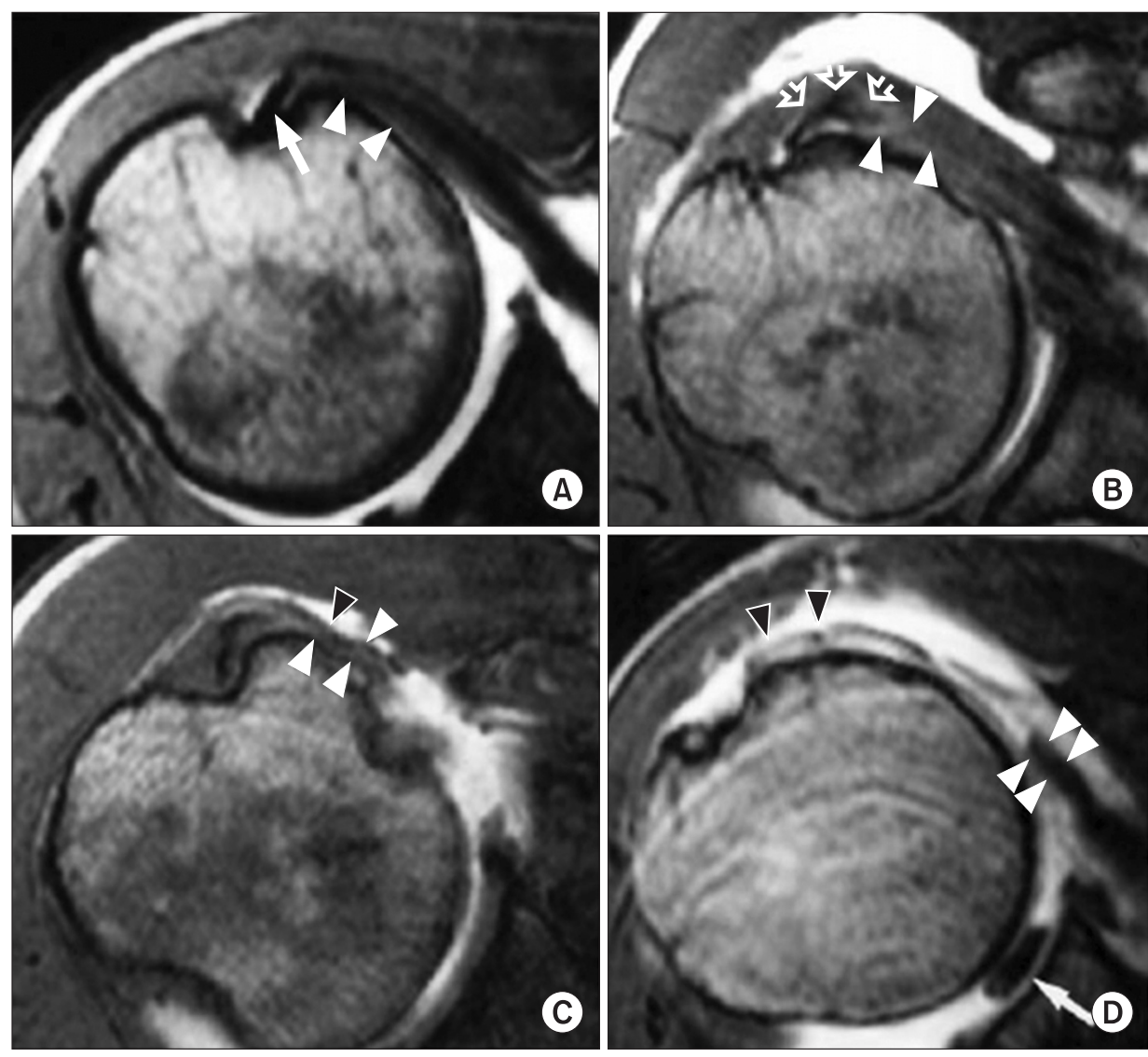

Fig. 1. MRI classification for subscapularis tendon tear by Pfirrmann. (A) Grade 0: Normal subscapularis tendon. (B) Grade 1: Slight fraying and signal alteration in the cranial part of the tendon. (C) Grade 2: Tear of the cranial $3 / 4$ of the subscapularis tendon. (D) Grade 3: Complete tear of the subscapularis tendon.
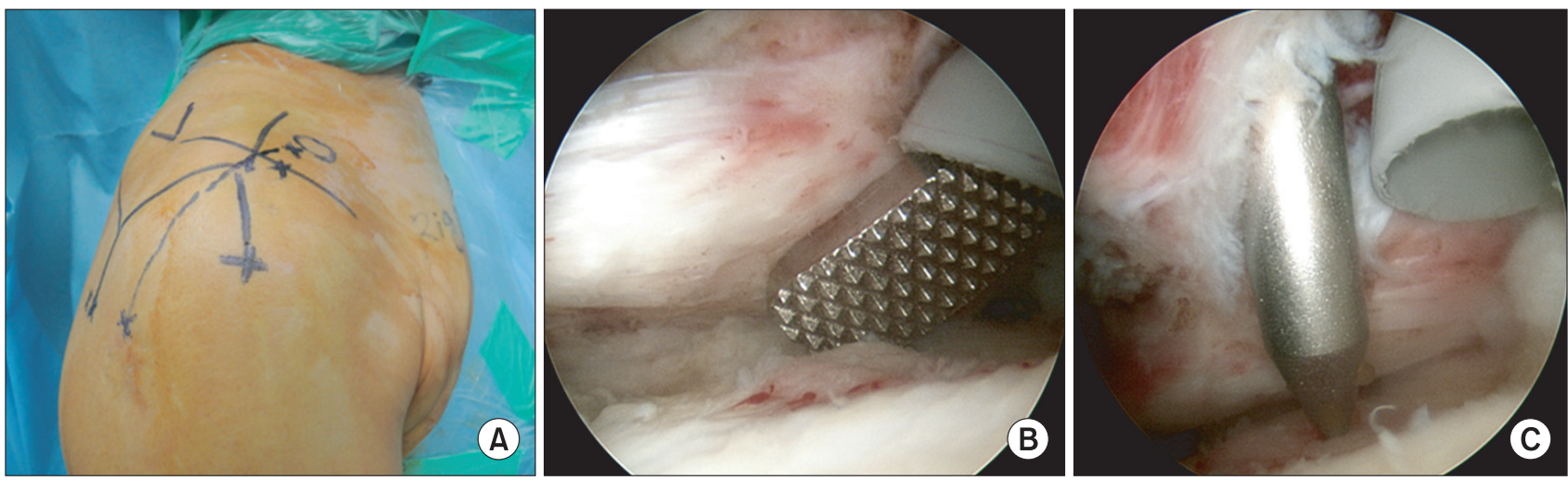

Fig. 2. (A) The posterior portal for the subscapularis tendon repair is located on the medial point of the conventional posterior portal. (B) The footprint of the subscapularis tendon was prepared. (C) The anchor guide for the suture anchor is positioned through the subclavian portal. 
addition, the integrity of cuff status was assessed by ultrasonographic examination at 6 months and at 1 year after operation. The statistical data were compared and analyzed using PASW Statics version 18.0 (IBM corporation).

\section{Surgical Procedure}

We followed the surgical procedure of our previous studies. After the patient was anaesthetized by interscalene block, they were put into a beach chair position with their neck slanted backwards. In this position, the OP drape was placed to expose the affected part of the shoulder and collar bone. First, the posterior portal was made on the inner side $(0.5 \mathrm{~cm})$ of the conventional portal site to examine the glenohumeral joint and the footprint of the subscapularis tendon. When examining the footprint of the subscapularis tendon, 70 degree or 30 degree arthroscopes were used interchangeably depending on the situation. Further, as in previous operations using the beach chair, the footprint was sufficiently examined by internally rotating the patients' arms (Fig. 2).

Following the examination of the joint, the anterosuperior portal was made using the out-in technique. Then, the subcla- vian portal was made on the inner side $(1-2 \mathrm{~cm})$ of the anterosuperior portal using an 18G needle. Through the subclavian portal, the exact location of the subscapularis tear was identified (Fig. 3).

To identify the severity of the subscapularis tendon tear, the damage level and instability of the long head of the bicep tendon and the bicep pulley were assessed. Afterwards, the hemorrhagic part of the footprint in the bony insertion of the lesser tuberosity was exposed through debridement with a file. Through the subclavian portal and then onto this bony insertion, an anchor punch was placed at an appropriate angle, upon which a biosuture anchor was inserted. Similarly, through the subclvian portal and landing through the inner part of the subscapularis tendon, the first limb of the interwave suture loaded with No.1 PDS suture was passed. This limb was also ligated with the suture of the suture anchor, thus allowing the suture to pass through the subscapularis tendon. Using this switching technique, the second limb of the interwave suture was passed through the anterolateral part of the subscapularis tendon.

For the ligation, the interwave suture was passed through the anterosuperior portal and first ligated with the outer part of
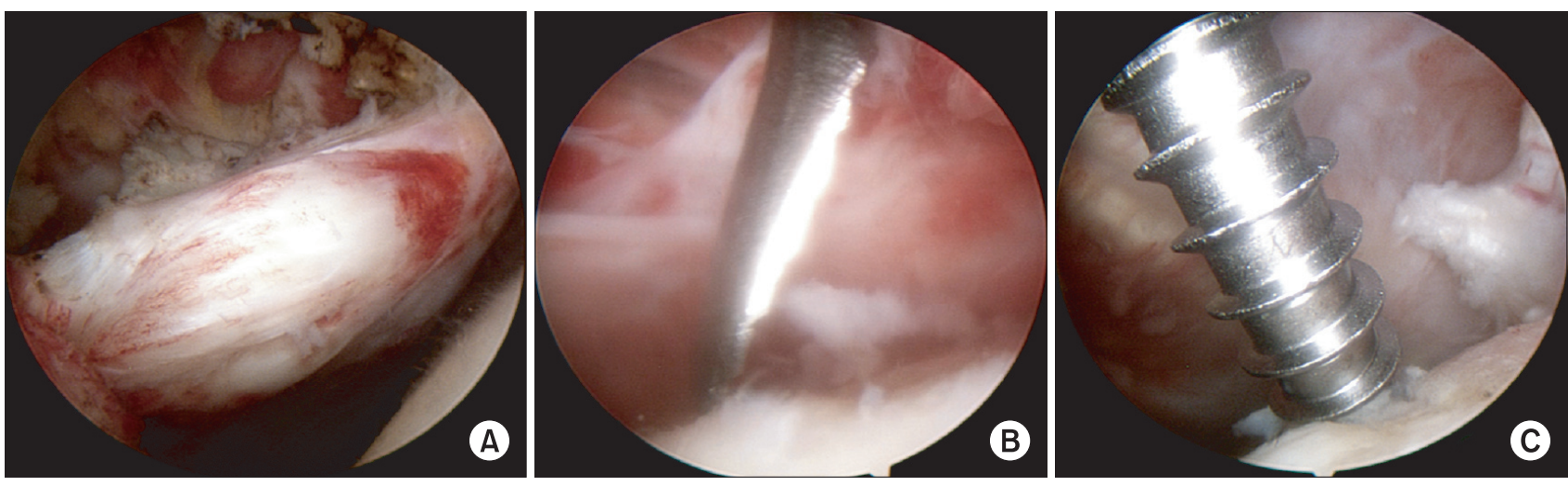

Fig. 3. (A) Identification of the subscapularis tear through the posterior portal. (B) Preparation of the footprint through the anterosuperior portal. (C) An anchor punch for the biosuture anchor was passed through the subclavian portal at an appropriate insertion angle.
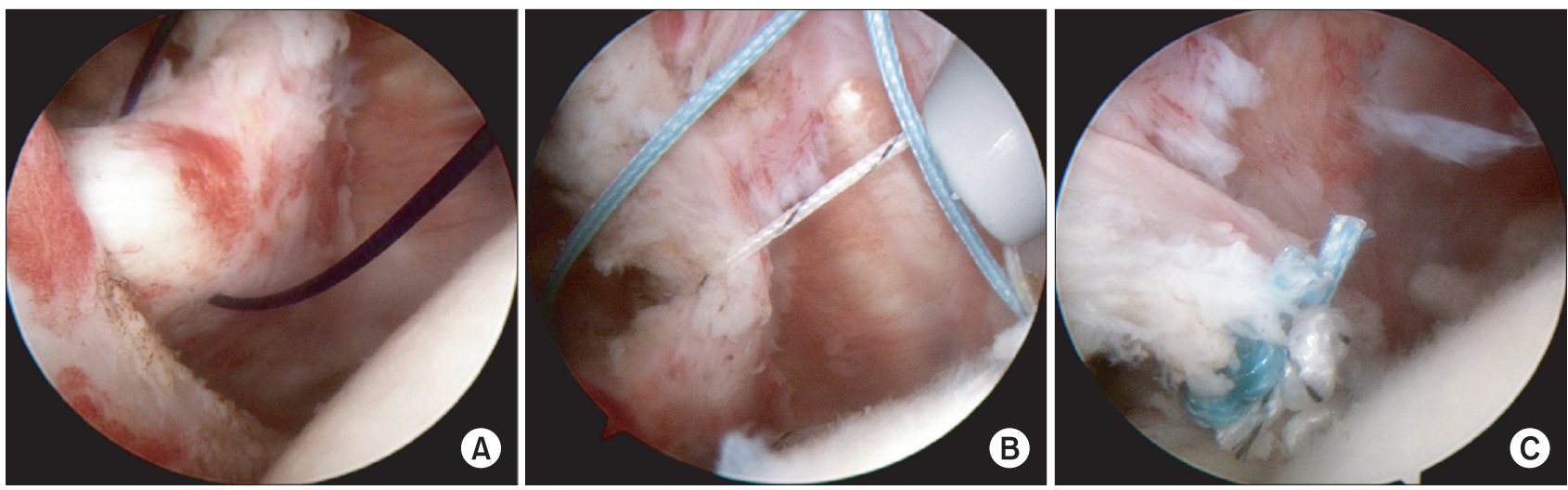

Fig. 4. (A) PDS was inserted through the subclavian portal and passed through the subscapularis tendon. (B) Both limbs of the suture anchor were passed through the tendon using a switching technique. (C) Completion of subscapularis tendon repair. 
the suture anchor, and then the inner part so that the 2 links of the suture did not interlink. After repair, the state of repair was assessed by internal and external rotation of the forearm at the neutral state (Fig. 4). As in previous studies, we used just one suture anchor. The average operation time was 52 minutes. Of the 18 cases, 2 cases had complete tear in the long head of the bicep tendon, and 3 cases had partial tear for which arthroscopic tenodesis was performed.

\section{Physiotherapy after Surgery}

After surgery an external rotation support was placed. This support was placed when the arm was at the neutral state with a slight internal rotation, and also took into account the recovery state of the repair. Physiotherapy was introduced gradually at various points after surgery and from a low intensity. At one day after operation, weight exercise was started and passive elevation exercise was only added when edema reduced. At 4-weeks, the external rotation support was removed and passive elevation exercise continued whilst maintaining the armrest. However, elevation through external rotation beyond the neutral state was limited until 6 weeks. At 6 weeks, the armrest was removed and active assistive exercise was started, which consists of the whole motion range including external rotation. At 8 weeks, active muscle strength training was started. At 3-4 months, patients were allowed to return to normal routine and do light exercise. Finally, at 6 months, patients were allowed to return to work and exercise to the full intensity from before the operation.

\section{Results}

The initial average range of motion in forward flexion, external rotation, and internal rotation were $160^{\circ}, 50^{\circ}$ and $\mathrm{L} 4$, respectively. These range of motions changed to $160^{\circ}, 52^{\circ}$ and $\mathrm{T} 12$, respectively at the 1-year follow-up period. Whilst the changes in forward flexion and external rotation are limited, the improvements in internal rotation is significant $(p<0.05)$

Table 1. ROM Improvement

\begin{tabular}{|c|c|c|c|c|c|c|c|c|c|}
\hline & \multirow{2}{*}{$\begin{array}{l}\text { Initial } \\
\text { Mean }\end{array}$} & \multicolumn{2}{|c|}{3 Month } & \multicolumn{2}{|c|}{6 Month } & \multicolumn{2}{|c|}{1 Year } & \multicolumn{2}{|c|}{ Final (35 months) } \\
\hline & & Mean (Diff S.D) & $p$-value & Mean (Diff S.D) & $p$-value & Mean (Diff S.D) & $p$-value & Mean (Diff S.D) & $p$-value \\
\hline $\mathrm{FF}$ & 160 & $135(30.53)$ & 0.0049 & $153.32(22.75)$ & 0.230 & $159(19.54)$ & 0.90 & $161.1(17.45)$ & 0.7964 \\
\hline ER & 50 & $40.3(10.64)$ & 0.0136 & $48.6(13.48)$ & 0.740 & $51(10.60)$ & 0.48 & $52.5(12.86)$ & 0.4908 \\
\hline $\mathrm{IR}^{*}$ & 1.82 & $2.54(2.02)$ & 0.278 & $3.54(2.47)$ & 0.021 & $5.71(2.47)$ & 0.00017 & $5.54(2.56)$ & 0.000 \\
\hline
\end{tabular}

$\mathrm{IR}^{\star}$ score: buttock $=0, \mathrm{~L} 5=1, \mathrm{~L} 4=2, \ldots, \mathrm{T} 8=10$.

Table 2. Functional Outcome

\begin{tabular}{|c|c|c|c|c|c|c|c|c|c|}
\hline & \multirow{2}{*}{$\begin{array}{c}\text { Initial score } \\
\text { (average) }\end{array}$} & \multicolumn{2}{|c|}{3 Month } & \multicolumn{2}{|c|}{6 Month } & \multicolumn{2}{|c|}{1 Year } & \multicolumn{2}{|c|}{ Final (35 months) } \\
\hline & & Score (Diff S.D) & $p$-value & Score (Diff S.D) & $p$-value & Score (Diff S.D) & $p$-value & Score (Diff S.D) & $p$-value \\
\hline ASES & 60 & $67(15.13)$ & 0.1856 & $77(16.14)$ & 0.0021 & $86(15.64)$ & 0.00002 & $84(15.27)$ & 0.000 \\
\hline ULCA & 26 & $27(4.43)$ & 0.4815 & $31(5.47)$ & 0.0043 & $32(6.1)$ & 0.0001 & $34(5.57)$ & 0.0000 \\
\hline Constant & 65 & $74(15.81)$ & 0.5498 & $75(14.23)$ & 0.042 & $79(14.33)$ & 0.002364 & $84(14.12)$ & 0.0000 \\
\hline KSS & 67 & 74 (12.59) & 0.03979 & 83 (11.98) & 0.0005 & $88(11.22)$ & 0.0000 & $92(11.09)$ & 0.0000 \\
\hline
\end{tabular}

Table 3. Effect of Associated LHB Lesion

\begin{tabular}{|c|c|c|c|c|c|}
\hline & \multicolumn{2}{|c|}{ LHB lesion (+) } & \multicolumn{2}{|c|}{ LHB lesion (-) } & \multirow[b]{2}{*}{$p$-value } \\
\hline & Preoperative score & $\begin{array}{l}\text { Post operative score } \\
\text { (Last follow-up) }\end{array}$ & Preoperative score & $\begin{array}{l}\text { Post operative score } \\
\text { (Last follow-up) }\end{array}$ & \\
\hline ASES & 54 & 100 & 70 & 96 & 0.375 \\
\hline ULCA & 25 & 34 & 30 & 33 & 0.056 \\
\hline Constant & 72 & 74 & 66 & 74 & 0.968 \\
\hline KSS & 68 & 90 & 72 & 91 & 0.375 \\
\hline
\end{tabular}



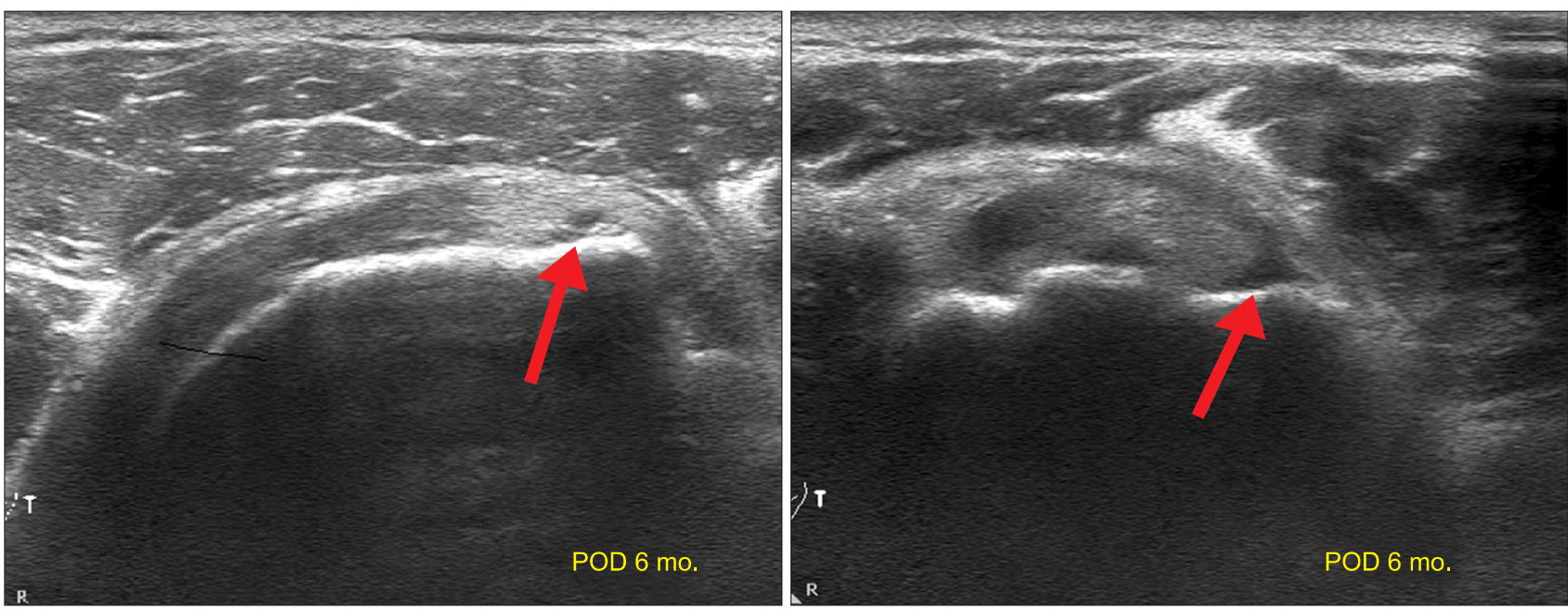

Fig. 5. Post-operative ultrasonographic examination revealed partially torn subscapularis tendon in both longitudinal and transverse scan.

(Table 1). The initial average functional scores were assessed by KSS, ASES, UCLA, and Constant scoring systems, which were $67,60,26$, and 65, respectively. The scores improved progressively with time. At 3 months after operation, the scores were $74,67,27$, and 74; at 6 months, 83, 77, 31, and 75; at 1 year, 88, 86, 32, and 79; and at the final follow-up of 35 months, 84, 92,34 , and 84 . The final scores are statistically significant and show a notable improvement from before the operation (Table 2). The belly press test, which came out all positive before the operation, came out negative in 17 cases (94\%) after the operation. Of the 5 cases with both isolated subscapularis tendon tear and long head of biceps tendon (LHB) lesion, 3 had additional arthroscopic tenodesis surgery. In these cases, no additional improvements were seen compared to the isolated subscapularis tendon tear (Table 3). In one case (5.5\%), re-rupture of the subscapularis tendon in the form of partial tear was identified by ultrasonographic examination at 6 months after operation (Fig. 5).

\section{Discussion}

Arthroscopic repair of the subscapularis tendon tear has several advantages over open treatment. The comparative advantages are that it is non-invasive and more aesthetic. At present, the most popular method of arthroscopic surgery is the use of anterosuperior and anterolateral portals at the lateral position as described by Burkhart et al. ${ }^{1,4}$ When carrying out this surgery, a good view of the footprint of the subscapularis tendon and an appropriate angle to insert the suture anchor is needed. To acquire a good view, a posterior lever push is carried out during which the upper part of the humerus is pushed and the lower part of the humerus is pulled simultaneously. To insert the suture anchor at an appropriate angle, the patient is put into a "hands on face" position which gives the "dead man" angle, the appropriate angle for insertion. The patient must wear goggles during this process for their safety. In contrast to the anterior portal, the posterior portal does not secure a good view of the footprint. In order to secure a better view, the author's of this paper inserted the posterior portal at a position approximately $0.5 \mathrm{~cm}$ medially than that of the conventional one. As a result, a better view of the footprint was secured during internal rotation of the patient arm in the beach chair position. In cases of partial tear, the footprint could be easily identified through adhesiolysis, and even in cases of cranial 3/4 tendon tear or complete tear, tendon proper could also be identified through adhesiolysis, because the tendon was relatively maintained with minimal retraction due to preserved comma sign. In these situations, the bicep pulley are usually well maintained, and thus can be used as anchor guides to the footprint.

There are several other advantages of using the subclavian portal for arthroscopic repair with the beach chair position. First, unlike the existing methods that use the anterosuperior and anterolateral portals, it does not damage the rotator cuff. Second, the subclavian portal gives a more ideal and safe angle of insertion of the suture anchor to the bony insertion in the lesser tuberosity, which is the anchor site of the subscapularis tendon. Lastly, it provides a larger working space, making it easier to thread the suture through the tendon. In our previous study, the authors used only one suture anchor because of the following differences in study backgrounds. First of all, in tendons with partial tears, one suture anchor was enough to fix the damaged parts and support the intact tendon. Secondly, even in tendons with cranial 3/4 tear or complete tear, two suture anchors are difficult to insert into the lateral part of the subscapularis tendon. Furthermore, because the muscles themselves are not damaged in these injuries, we anticipated tenodesis effect by fixing the tendon with one suture. Additionally, in tendons with complete tear, the repair site on footprint is inevitably displaced to the inner side. This medial displacement could be accentuated if 
two suture anchors are used in the upper and lower part of the footprint. As a result, we decided to use just one suture anchor in appropriate lateral position with a 6 week period of immobilization.

Several papers have reported their findings on the efficacy of the subclavian portal in arthroscopic surgery. Since their report on the arthroscopic repair of the rotator cuff, Nord et al. ${ }^{5,6)}$ further reported that the subclavian portal can also be used in the repair of the long head of the bicep tendons. ${ }^{7)}$ Daluga and Daluga. ${ }^{8)}$ also reported that the subclavian portal can be used to repair SLAP lesions. In our present study, we used the subclavian portal to repair subscapularis tendon tears and to find out its clinical efficacy. ${ }^{9)}$

Ultrasonograms were used to check the maintenance of the repaired subscapularis tendon. Although MRI imaging is commonly used for this purpose, ${ }^{7)}$ ultrasonograms can identify early changes, be used easily in outpatient clinics, and identify dynamic changes. ${ }^{10)}$ Ambacher and Holz ${ }^{11)}$ explained that the typical sign of the re-tear may be correlated to the decreased thickness of the tendon, which can be identified using static and dynamic images on both shoulder. On the other hand, Zehetgruber et al. ${ }^{12)}$ said that analyzing repair in this way is limited. In our study, there was one case of re-rupture (from complete tear before operation to partial tear after). This was identified by transverse and longitudinal scans of the subscapularis tendon as it was undergoing internal and external rotation. In both of these scans, we detected changes in the echo level of the attachment site of the tendon (Fig. 4), reflecting re-rupture.

In a study by Kim et al., ${ }^{2)} 29$ cases of subscapularis partial tear underwent arthroscopic repair. In 28 (96\%) of these cases, repair outcomes were above average and there was a decrease in the power of internal rotation (34\% to $4 \%$ ), showing a good clinical outcome. In a study by Lafosse et al., ${ }^{13)} 17$ cases of isolated subscapularis tendon tear underwent repair. Both the constant score and UCLA score improved, from 58\% to $96 \%$ and from 16 points to 32 points, respectively. Furthermore, the repair continued to be well maintained in 15 cases (88\%). Edward et al. ${ }^{14)}$ carried out 84 cases open treatments on subscapularis tendon. Of these surgeries, 48 cases were accompanied by tenodesis of the LHB tendon, 13 cases were accompanied by tenotomy, and 4 cases were accompanied with recentering of the tendon. According to their findings, the Constant score improved from 55 points to 79.5 points, thus treatment of the bicep tendon may be an effective supportive surgery. Maier et al. ${ }^{15)}$ also carried out recentering of the long head of the bicep tendon. They reported that in younger patients particularly, recentering can substitute for tenodesis and tenotomy.

In subscapularis tendon tear, the damage frequency of the LHB tendon is usually around $47 \%{ }^{16)}$ However, our study reports a lower damage frequency in the bicep tendon than that of previous reports. This is because our study involves 12 cases of isolated subscapularis tendon tear due to trauma, which means that extension injury and traumatic ruptures are key elements. These two elements have a relatively lower effect on the LHB tendon and the biceps pulley, which is liked to be the reason why we report a lower damage frequency in the bicep tendon. Improvements after surgery were similar. At 6 months after operation, the motion range of the internal rotation of the joint improved significantly from L4 to T11, whilst the range of the forward elevation and external rotation did not change significantly $(p>0.05)$ even at 1 year after operation. The motion range of the forward elevation and external rotation were 160 degrees and 50 degrees before surgery and 160 degrees and 52 degrees after surgery, respectively. Functional recovery, as assessed by KSS, ASES, UCLA, and Constant score systems, improved significantly at 1 year after surgery. Even at the final follow-up of 35 months, function improvement was statistically significant, again assessed by the same scoring systems. Similarly, the belly press test improved before and after the operation, from positive to negative in 17 cases (94\%).

To sum, we used the subclavian portal to repair isolated subscapularis tendon tear. The treatment was effective, partly due to the co-treatment of the tear in the long head of the bicep tendon. Furthermore, for full recovery of function, the treatment needed nearly up to 3 years of follow-up involving close observation and appropriate physiotherapy.

However, several limitations have been noted in this study. First is the inconsistent use of imaging techniques during the treatment period. Before the operation, MRI was used to analyze the initial state of the subscapularis tendon, whilst after the operation; ultrasonogram was used to analyze the recovery state. This inconsistency in analysis means that we cannot fully compare the images during tendon healing. Furthermore, we only obtained the ultrasound images up to 1 year after surgery, which means a lack of long-term follow-up.

To repair the isolated subscapularis tendon tear, we used the subclavian portal through which a suture anchor and interwave suture technique were used. This surgery method was effective because it provided an appropriate angle for the suture anchor, made sufficient space for repair and used just one suture anchor. Furthermore, ultrasonograms were effective to dynamically assess the recovery state of the tendon during follow-up.

\section{References}

1. Burkhart SS, Tehrany AM. Arthroscopic subscapularis tendon repair: Technique and preliminary results. Arthroscopy. 2002;18(5):454-63.

2. Kim SH, Oh I, Park JS, Shin SK, Jeong WK. Intra-articular repair of an isolated partial articular-surface tear of the subscapularis tendon. Am J Sports Med. 2005;33(12):1825-30.

3. Pfirrmann CW, Zanetti M, Weishaupt D, Gerber C, Hodler J. 
Subscapularis tendon tears: detection and grading at MR arthrography. Radiology. 1999;213(3):709-14.

4. Burkhart SS, Brady PC. Arthroscopic subscapularis repair: surgical tips and pearls A to Z. Arthroscopy. 2006;22(9):1014-27.

5. Nord KD, Mauck BM. The new subclavian portal and modified Neviaser portal for arthroscopic rotator cuff repair. Arthroscopy. 2003;19(9):1030-4.

6. Nord KD, Smith GB, Mauck BM. Arthroscopic biceps tenodesis using suture anchors through the subclavian portal. Arthroscopy. 2005;21(2):248-52.

7. Ozbaydar MU, Altan E, Esenyel CZ, Yalaman O. Surgical treatment of isolated post-traumatic subscapularis tendon ruptures. Acta Orthop Traumatol Turc. 2006;40(3):214-9.

8. Daluga DJ, Daluga AT. Single-portal SLAP lesion repair. Arthroscopy. 2007;23(3):321.

9. Choi CH, Kim SK, Chang IW, Kim SS. Subclavian portal approach for isolated subscapularis tendon tear: technical note. J Korean Shoulder Elb Soc. 2009;12(2):221-5.

10. Iovane A, Midiri M, Bartolotta TV, Carcione A, Lagalla R. Normal anatomy and pathological conditions of subscapular muscle: US findings compared with surgery. Radiol Med.

$$
\text { 2001;101(4):260-4. }
$$

11. Ambacher T, Holz U. Ruptures of the subscapular tendon. A diagnostic problem? Unfallchirurg. 2002;105(5):486-91.

12. Zehetgruber $H$, Lang $T$, Wurnig $C$. Distinction between supraspinatus, infraspinatus and subscapularis tendon tears with ultrasound in 332 surgically confirmed cases. Ultrasound Med Biol. 2002;28(6):711-7.

13. Lafosse L, Jost B, Reiland Y, Audebert S, Toussaint B, Gobezie R. Structural integrity and clinical outcomes after arthroscopic repair of isolated subscapularis tears. J Bone Joint Surg Am. 2007;89(6):1184-93.

14. Edwards TB, Walch G, Sirveaux F, et al. Repair of tears of the subscapularis. J Bone Joint Surg Am. 2005;87(4):725-30.

15. Maier D, Jaeger M, Suedkamp NP, Koestler W. Stabilization of the long head of the biceps tendon in the context of early repair of traumatic subscapularis tendon tears. J Bone Joint Surg Am. 2007;89(8):1763-9.

16. Beall DP, Williamson EE, Ly JQ, et al. Association of biceps tendon tears with rotator cuff abnormalities: degree of correlation with tears of the anterior and superior portions of the rotator cuff. AJR Am J Roentgenol. 2003;180(3):633-9. 